\title{
APPLICATION OF SOCIAL COGNITIVE THEORY ON FACTORS AFFECTING COMPLETENESS OF CHILD IMMUNIZATION: EVIDENCE FROM PACITAN, EAST JAVA
}

\author{
Puspita Mayangsari Prabowo'), Setyo Sri Rahardjo²), \\ Eti Poncorini Pamungkasari²) \\ 1)Masters Program in Public Health, Universitas Sebelas Maret \\ 2)Faculty of Medicine, Universitas Sebelas Maret
}

\begin{abstract}
Background: Every infant is required to receive complete basic immunization before the age of one year. According to UNICEF, around 19.4 million infants do not receive complete basic immunization. The purpose of this study was to examine factors affecting completeness of child immunization using Social Cognitive Theory.

Subjects and Method: A cross sectional study was carried out at 25 integrated health posts (posyandu) in Pacitan, East Java, Indonesia. A sample of 200 infants aged 12-23 months was selected by simple random sampling. The dependent variable was basic immunization completeness. The independent variables were intention, knowledge, self-efficacy, outcome expectation, vicarious learning, family support, health personnel support, and social capital. The data were analyzed by a multiple logistic regression rin on Stata 13.

Results: Complete immunization status increased with high knowledge $(\mathrm{OR}=13.39$; 95\% CI= 2.12 to $84.39 ; \mathrm{p}=0.006)$, strong self-efficacy $(\mathrm{OR}=6.05 ; 95 \% \mathrm{CI}=1.29$ to $28.35 ; \mathrm{p}=0.022)$, positive outcome expectation $(\mathrm{OR}=15.20 ; 95 \% \mathrm{CI}=2.75$ to 83.89; $\mathrm{p}=0.002)$, vicarious learning $(\mathrm{OR}=4.30 ; 95 \% \mathrm{CI}=0.81$ to $22.67 ; \mathrm{p}=0.085)$, strong family support $(\mathrm{OR}=6.36 ; 95 \% \mathrm{CI}=1.30$ to $31.11 ; \mathrm{p}=0.022)$, strong health personnel support $(\mathrm{OR}=20.38 ; 95 \% \mathrm{CI}=3.16$ to $131.36 ; \mathrm{p}=$ o.002), strong intention ( $\mathrm{OR}=43.52 ; 95 \% \mathrm{CI}=4.20$ to $450.515 ; \mathrm{p}=0.002)$, and strong social capital $(\mathrm{OR}=4.41 ; 95 \% \mathrm{CI}=0.98$ to $19.85 ; \mathrm{p}=0.053)$.

Conclusion: Complete immunization status increases with high knowledge, strong selfefficacy, positive outcome expectation, vicarious learning, strong family support, strong health personnel support, strong intention, and strong social capital.
\end{abstract}

Keywords: complete immunization status, social capital, Social Cognitive Theory

\section{Correspondence:}

Puspita Mayangsari Prabowo. Masters Program in Public Health, Universitas Sebelas Maret. Jl. Ir. Sutami 36A, Surakarta 57126, Central Java. Email: mayangsari37.pm@gmail.com. Mobile: +6287859294881 . 